\title{
Efficient Simulation of Epistatic Interactions in Case-Parent Trios
}

\author{
Qing Li $^{\mathrm{a}}$ Holger Schwender ${ }^{\mathrm{b}}$ Thomas A. Louis ${ }^{c}$ M. Daniele Fallin ${ }^{d}$ \\ Ingo Ruczinskic
}

${ }^{a}$ Statistical Genetics Section, National Human Genome Research Institute, National Institutes of Health, Baltimore, Md., USA; b Mathematical Institute, Heinrich Heine University Düsseldorf, Düsseldorf, Germany; Departments of 'Biostatistics, and dEpidemiology, Johns Hopkins Bloomberg School of Public Health, Baltimore, Md., USA

\section{Key Words}

Case-parent trios $\cdot$ Interactions $\cdot$ Single nucleotide

polymorphisms · Haplotypes

\begin{abstract}
Statistical approaches to evaluate interactions between single nucleotide polymorphisms (SNPs) and SNP-environment interactions are of great importance in genetic association studies, as susceptibility to complex disease might be related to the interaction of multiple SNPs and/or environmental factors. With these methods under active development, algorithms to simulate genomic data sets are needed to ensure proper type I error control of newly proposed methods and to compare power with existing methods. In this paper we propose an efficient method for a haplotype-based simulation of case-parent trios when the disease risk is thought to depend on possibly higher-order epistatic interactions or gene-environment interactions with binary exposures.
\end{abstract}

Copyright $\odot 2013$ S. Karger AG, Basel
() 2013 S. Karger AG, Basel

0001-5652/13/0751-0012\$38.00/0

\section{Introduction}

Statistical approaches to evaluate higher-order interactions between single nucleotide polymorphisms (SNPs), or between SNPs and environmental variables, are critical for analyzing complex diseases, as higher susceptibility is likely to be related to the interaction of multiple SNPs and/or environmental factors. The effect sizes seen in complex diseases are typically very small, and therefore the power to detect those small effect sizes can crucially depend on whether methods to simultaneously investigate SNPs and environmental variables are employed, i.e. whether or not such interactions are directly assessed. This creates statistical challenges in the analysis of how SNPs and environmental variables relate to the disease outcome, since the number of possible interactions between genetic markers and the environmental factors is immense. To address this issue, many tools from the statistical and machine learning literature, developed to deal with high-dimensional search spaces, have been applied to multi-marker SNP data, e.g. neural networks [1-3], random forests [4-7], and various other methods based on partitions, trees, and splines, and ensembles of base learners [8-10]. Some approaches to delineate higher-order interactions were specifically developed for SNP data,

\section{KARGER}

E-Mail karger@karger.com

www.karger.com/hhe
Ingo Ruczinski

Department of Biostatistics

Johns Hopkins Bloomberg School of Public Health

Baltimore, MD 21205 (USA)

E-Mail ingo@jhu.edu 
such as the multifactor dimensionality reduction techniques [11-15], the restricted partition method [16, 17], and logic regression [18-20].

The above-cited methods are typically employed in case-control or cohort studies, relating genotypes to binary, ordinal, or numeric phenotypes. In addition to population-based designs, family-based studies offer an appealing alternative, since these designs are robust against population substructure, and allow for the assessment of linkage and association [21-24]. Arguably, the simplest and most prominent test is the transmission disequilibrium test (TDT), a completely nonparametric approach applicable to trios of parents plus an affected offspring [25]. Many extensions to the TDT have been proposed, e.g. allowing for multi-allelic and haplotype-based tests, general pedigrees, missing data, quantitative traits, many using parametric approaches (see for example Laird and Lange [26] for a comprehensive review).

Much thought and effort was provided by the community to devise methods to detect gene-gene and gene-environment interactions in family data, particularly for nuclear families, and most prominently among those for parent-affected-child trios [27-36]. These approaches quantify the statistical significance of candidate interactions under investigation, but they do not provide algorithms to allow for the actual search of SNP-SNP interactions. The task to search for and the assessment of higherorder interactions can be achieved by two approaches. MDR-PDT [37] merges the genotype-pedigree disequilibrium test [38] with the multifactor dimensionality reduction technique [12]: multilocus genotypes are pooled into high-risk and low-risk groups, sequentially reducing the dimensionality of the predictors. Trio-logic regression [39] is an extension of the logic regression methodology [18-20] and the genotypic TDT [40,41], employing probabilistic search algorithms (simulated annealing) to detect and assess higher-order interactions in trios with affected probands. The trio data are set up for conditional logistic regression with a 1:3 matching ratio for the case genotypes versus three possible Mendelian realizations given the parents ('pseudo-controls'), taking the linkage disequilibrium (LD) structure in the genotype data into account.

With these methods for the analysis of genomic trio data under active development, algorithms to simulate such data sets are critical, to ensure proper type I error control of newly proposed methods, and to compare power with existing methods. With substantial advancement of computational power in the last 20 years, simulation methods of genetic family data have become avail- able and now play a prominent role in statistical genetics. A vast literature exists on methods that can simulate large and complex evolutionary processes, starting with the founders of the population and creating pedigrees over dozens of generations, working forward in time [42-50], and computationally efficient coalescent theory-based algorithms that work backwards in time [51-61]. Simulations of triads of parents and offspring are typically simpler, in particular under the assumptions of random mating and Hardy-Weinberg equilibrium. For complicated models of disease risk, case-parent trios are often simulated prospectively by randomly sampling parental haplotypes from the population, generating diplotypes for an offspring, and simulating the child's affection status under the genetic model using the genotypes at the disease loci [62]. The case-parent trios are then randomly selected from the subset of triads with affected probands. While easily implemented, this approach is computationally inefficient, and in particular under the assumptions of rare diseases and/or small genetic effects might require an exorbitant number of trios to be simulated. For some disease-risk models, however, the conditional joint distribution of haplotypes in parents and affected proband has a closed-form solution (see for example table 2 in Ainsworth et al. [63]), and thus the genotypes for case-parent trios can be sampled directly from this distribution, yielding a much more efficient sampling approach. In this paper, we propose a novel and efficient retrospective sampling method for a haplotype-based simulation of caseparent trios, when the disease risk is thought to depend on epistatic interactions, or gene-environment interactions with binary exposures.

\section{Methods}

We describe an efficient approach to simulate case-parent trios, a key ingredient to validate new methods and software packages. For family data, simulations based on haplotypes and mating tables very often result in computationally intractable problems. We first show that a naive enumeration of mating patterns quickly results in unworkable dimensionalities, and we introduce an alternative that makes the required computations feasible. The following simulation methods have been implemented in the function trio.sim() in the $\mathrm{R}$ package trio.

\section{Two Risk-Group Model}

We assume two risk groups in the population defined by some genotype pattern $G$, and we assume that the probability of disease $p$ is given via the log-odds as

$$
\log \left(\frac{p}{1-p}\right)=\alpha+\beta I_{G},
$$

Efficient Simulation of Epistatic Interactions in Case-Parent Trios
Hum Hered 2013;75:12-22

DOI: $10.1159 / 000348789$ 
where $I$ is the indicator function, and $\alpha$ and $\beta$ are some fixed parameters. We encode the genotype patterns using Boolean combinations of SNPs in dominant and recessive coding. This allows for a convenient specification of epistatic interactions, such as doublepenetrance models (fig. 1), but also of more complicated higherorder biological interactions of interest. Further, binary environmental factors can easily be included in the interaction term. For example, a statement such as 'the odds of disease for smokers who have at least one variant allele at both SNP 7 and SNP 12 are three times higher compared to the rest of the population' can easily be encoded.

To simplify notation, we follow Weinberg et al. [64] and use the letters $F, M$, and $C$ to represent the haplotype pairs (diplotypes) of the father, the mother and the child. We refer to the joint probability distribution of $F, M$, and $C$ as the mating table. Further, we use the letter $D$ to indicate an affected proband. To simulate case-parent trios, we therefore need to specify $P(F, M, C \mid D)$ for each haplotype block (assuming independence between blocks).

For haplotype blocks that do not contain a locus involved in the genetic signal that defines the high-risk group in equation 1 , the haplotype frequencies in the trios do not depend on the disease status of the child. Thus, we have $P(F, M, C \mid D)=P(F, M, C)$ for a haplotype block that does not contain information about the disease risk. Further, $P(F, M, C)=P(M, F) \times P(C \mid M, F)=P(M) \times$ $P(F) \times P(C \mid M, F)$, under the assumption of random mating. Thus, for any block that does not contain information about the disease risk, we can sample the genotypes for the trios by randomly selecting two haplotypes for each parent using the population frequencies, and generate the proband's diplotype from the parents' haplotype pairs assuming independent segregation.

To generate the trio genotypes for the blocks that do contain information about disease risk, we have to take into account that the haplotype frequencies in those blocks are different from the population at large, if we condition on having an affected proband. However, enumerating the entire mating table and calculating all these probabilities is prohibitive. Assume for example that there are 3 blocks with loci that contribute to the disease risk with 5 haplotypes each. Thus, there are $5^{3}=125$ possible haplotype sets for a subject, resulting in $125 \times(125+1) / 2=7,875$ possible diplotypes for these 3 blocks combined. For a pair of parents we have $7,875 \times$ $(7,875+1) / 2 \approx 31$ million haplotype-pair combinations. Thus, we pursue a different strategy that avoids generating the whole mating table. We first sample the haplotype pairs for affected probands, and then sample the parents' haplotype pairs given the proband's diplotype. In particular, we use

$$
P(F, M, C \mid D)=P(F, M \mid C, D) \times P(C \mid D)=P(F, M \mid C) \times P(C \mid D) .
$$

That is, we first sample a diplotype for an affected proband, and then sample the parents given the affected proband. We can avoid extensive enumerations by taking advantage of the fact that there are only two risk groups. The procedure is best explained in an example. Assume that there are 2 haplotype blocks with 2 and 3 loci, respectively, that specify the disease risk (table 1) as follows:

$$
\log \left(\frac{p}{1-p}\right)=\alpha+\beta \times I\left[\left(\mathrm{SNP}_{1}^{R} \wedge \overline{\mathrm{SNP}_{3}^{D}}\right) \vee \mathrm{SNP}_{5}^{R}\right] .
$$

Thus, subjects with 2 variant alleles at SNP 5 (the third locus in block 2) are at higher risk as are subjects with 2 variant alleles at SNP 1 (the first locus in block 1) and subjects with no variant al-

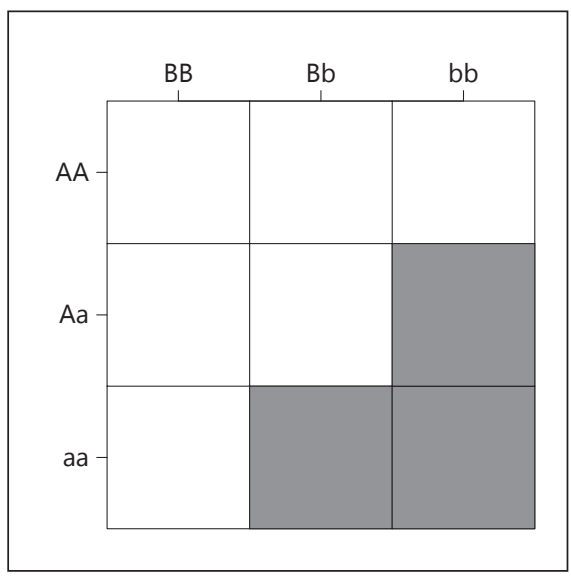

Fig. 1. Illustration of a double-penetrance model, assuming that disease risk depends on the interaction between SNPs. Common alleles for markers A and B are denoted by capital letters, the variant alleles using small letters. Subjects with 2 variant alleles at 1 SNP and at least 1 variant allele at the other SNP are at higher risk for disease. Using dominant and recessive coding, the grey region can be described as $\left(\mathrm{SNP}_{A^{\mathrm{D}}} \wedge \mathrm{SNP}_{B^{\mathrm{R}}}\right) \vee\left(\mathrm{SNP}_{B^{\mathrm{D}}} \wedge \mathrm{SNP}_{A^{\mathrm{R}}}\right)$, where $\wedge$ and $\vee$ denote the Boolean operators 'and' and 'or', respectively.

Table 1. An example of 2 haplotype blocks having lengths 2 and 3 with 3 and 4 possible haplotypes, respectively

\begin{tabular}{llll}
\hline Block & Index & Haplotype & Frequency \\
\hline 1 & 1 & 11 & $f_{1 \mid 1}$ \\
1 & 2 & 21 & $f_{2 \mid 1}$ \\
1 & 3 & 22 & $f_{3 \mid 1}$ \\
\hline 2 & 1 & 121 & $f_{1 \mid 2}$ \\
2 & 2 & 122 & $f_{2 \mid 2}$ \\
2 & 3 & 111 & $f_{3 \mid 2}$ \\
2 & 4 & 222 & $f_{4 \mid 2}$ \\
\hline
\end{tabular}

In the above notation, $f_{i j}$ refers to the $i$-th haplotype in block $j$.

leles at SNP 3 (the first locus in block 2). We assume 3 possible haplotypes in block 1 , and 4 possible haplotypes in block 3 , resulting in 12 possibilities for a haplotype spanning both blocks (table 2 ). Therefore, there are $12 \times(12+1) / 2=78$ possible diplotypes in our population. Out of those 78 diplotypes, 22 have the risk genotype combination as specified above (table 3 ), and we tabulate the haplotype pairs accordingly in high-risk and low-risk diplotypes (table 4). For computational efficiency and convenience, we also differentiate between identical and non-identical haplotypes in a diplotype (denoted as strata $\mathrm{HR}_{1}, \mathrm{HR}_{2}, \mathrm{LR}_{1}$, and $\mathrm{LR}_{2}$ ), and further stratify by the possible indices of the first haplotype we will sample in a pair. Note that this step needs to be carried out only once, when the simulation is initialized. 
Table 2. The 12 possible haplotypes across 2 blocks with 3 and 4 haplotypes, respectively

\begin{tabular}{cllllll}
\hline \multirow{2}{*}{ Index } & \multicolumn{2}{l}{ Haplotype index } & & \multicolumn{2}{l}{ Haplotype } & Frequency \\
& block 1 & block 2 & & block 1 & block 2 & \\
\hline 1 & 1 & 1 & 11 & 121 & $P\left(H_{1}\right)=f_{1 \mid 1} \times f_{1 \mid 2}$ \\
2 & 2 & 1 & 21 & 121 & $P\left(H_{2}\right)=f_{2 \mid 1} \times f_{1 \mid 2}$ \\
3 & 3 & 1 & 22 & 121 & $P\left(H_{3}\right)=f_{3 \mid 1} \times f_{1 \mid 2}$ \\
\hline 4 & 1 & 2 & 11 & 122 & $P\left(H_{4}\right)=f_{1 \mid 1} \times f_{2 \mid 2}$ \\
5 & 2 & 2 & 21 & 122 & $P\left(H_{5}\right)=f_{2 \mid 1} \times f_{2 \mid 2}$ \\
6 & 3 & 2 & 22 & 122 & $P\left(H_{6}\right)=f_{3 \mid 1} \times f_{2 \mid 2}$ \\
\hline 7 & 1 & 3 & 11 & 111 & $P\left(H_{7}\right)=f_{1 \mid 1} \times f_{3 \mid 2}$ \\
8 & 2 & 3 & 21 & 111 & $P\left(H_{8}\right)=f_{2 \mid 1} \times f_{3 \mid 2}$ \\
9 & 3 & 3 & 22 & 111 & $P\left(H_{9}\right)=f_{3 \mid 1} \times f_{3 \mid 2}$ \\
\hline 10 & 1 & 4 & 11 & 222 & $P\left(H_{10}\right)=f_{1 \mid 1} \times f_{4 \mid 2}$ \\
11 & 2 & 4 & 21 & 222 & $P\left(H_{11}\right)=f_{2 \mid 1} \times f_{4 \mid 2}$ \\
12 & 3 & 4 & 22 & 222 & $P\left(H_{12}\right)=f_{3 \mid 1} \times f_{4 \mid 2}$ \\
\hline
\end{tabular}

The haplotype frequencies are derived from the frequencies in table 1 .

To reflect the diplotype distribution among affected probands, we have to consider the probability of disease given the genotype, and we note that $P(C \mid D) \propto P(D \mid C) \times P(C)$. Moreover, for any 2 children $C_{1}$ and $C_{2}$ within the same diplotype stratum as defined in table 4 , we have $P\left(D \mid C_{1}\right)=P\left(D \mid C_{2}\right)$. Thus, if $S$ denotes the union of all diplotypes within a stratum, we have

$$
P(S \mid D) \propto P_{S}(D) \times P(S),
$$

where $P_{S}(D)$ is the probability of disease, equal for every element in $S$. Thus, we can use the set of probabilities $P(S \mid D)$ as the sampling probabilities to pick a stratum in the initial step in the sampling procedure. We then sample a haplotype (index $i$ ) using the haplotypepair probabilities within the selected stratum indicated in table 4, and then pick a second haplotype (index $j$ ) from the set of permissible haplotypes, using the haplotype frequencies from this set.

With the diplotype of the affected proband available, the joint diplotype distribution for the parents $P(F, M \mid C)$ is rather straightforward. For computational efficiency, we again divide the joint parental distributions into strata (table 5). We distinguish between cases where the proband's haplotype pairs are identical or where they are not. The strata's probabilities are then used to sample the actual haplotype pairs for the parents (table 6). Two considerations are important in the calculation of the sampling probabilities. Since there is no ordering among the parents, the joint probability for a non-identical pair of diplotypes is multiplied by the factor 2 . Further, depending on the parents' haplotype combinations, the number of distinct diplotypes among the 4 'Mendelian' children can be 1,2 , or 4 . To calculate the correct sampling probabilities, these issues are taken into consideration by multiplying the parents' diplotype probabilities with the respective factors (table 6). In summary, given the child's diplotype, we first derive the strata's probabilities of parent's diplotypes (table 5), and then calculate
Table 3. The diplotypes and genotypes for the risk group, assuming the defining interaction is $\left(\mathrm{SNP}_{1}^{R} \wedge \mathrm{SNP}_{3}^{D}\right) \vee \mathrm{SNP}_{5}^{R}$

\begin{tabular}{llll}
\hline Pair & Haplotype 1 & Haplotype 2 & Genotype \\
\hline$(1,2)$ & 11121 & 21121 & $12-11-11-22-11$ \\
$(1,3)$ & 11121 & 22121 & $12-12-11-22-11$ \\
$(1,7)$ & 11121 & 11111 & $11-11-11-12-11$ \\
$(1,8)$ & 11121 & 21111 & $12-11-11-12-11$ \\
$(1,9)$ & 11121 & 22111 & $12-12-11-12-11$ \\
\hline$(2,3)$ & 21121 & 22121 & $22-12-11-22-11$ \\
$(2,7)$ & 21121 & 11111 & $12-11-11-12-11$ \\
$(2,8)$ & 21121 & 21111 & $22-11-11-12-11$ \\
$(2,9)$ & 21121 & 22111 & $22-12-11-12-11$ \\
$(3,7)$ & 22121 & 11111 & $12-12-11-12-11$ \\
$(3,8)$ & 22121 & 21111 & $22-12-11-12-11$ \\
\hline$(3,9)$ & 22121 & 22111 & $22-22-11-12-11$ \\
$(7,8)$ & 11111 & 21111 & $12-11-11-11-11$ \\
$(7,9)$ & 11111 & 22111 & $12-12-11-11-11$ \\
$(8,9)$ & 21111 & 22111 & $22-12-11-11-11$ \\
$(1,1)$ & 11121 & 11121 & $11-11-11-22-11$ \\
\hline$(2,2)$ & 21121 & 21121 & $22-11-11-22-11$ \\
$(3,3)$ & 22121 & 22121 & $22-22-11-22-11$ \\
$(7,7)$ & 11111 & 11111 & $11-11-11-11-11$ \\
$(8,8)$ & 21111 & 21111 & $22-11-11-11-11$ \\
$(9,9)$ & 22111 & 22111 & $22-22-11-11-11$ \\
$(10,10)$ & 11222 & 11222 & $11-11-22-22-22$ \\
\hline & & & \\
\hline
\end{tabular}

Here, 1 indicates the minor (variant) allele, and 2 indicates the major allele. Therefore, subjects with 2 variant alleles at locus 5 (genotype 11) are at higher risk, as are subjects with both 2 variant alleles (genotype 11) at locus 1 and no variant alleles (genotype 22) at locus 3

the sampling probabilities for diplotype pairs for the parents (table 6). The general outline of the algorithm is shown in the online supplementary materials (for all online suppl. material, see www. karger.com/doi/10.1159/000348789).

\section{Multiple Loci Disease Model}

In the above derivations, we assumed that only two risk groups exist in the population (carriers and non-carriers). A convenient framework to extend this setting to a risk model that allows for additional risk loci beyond the epistatic interaction is the 'twolocus' genetic heterogeneity model [65], which assumes that the 'loci' affect the outcome independently. In addition to the specified interaction (in our case) taken as the first 'locus', the second 'locus' can be understood to simply represent all other disease loci that define disease risk in an additive model. Assuming that the two risk sets are independent causes of disease, we can then write the probability that an individual is affected as a function of the probabilities that the subject becomes affected through the respective loci:

$$
P(D \mid C, X)=1-[1-P(D \mid C)] \times[1-P(D \mid X)],
$$


Table 4. The haplotype pairs in a population are tabulated according to high-risk and low-risk diplotypes, as given in table 3

\begin{tabular}{|c|c|c|c|c|c|}
\hline Stratum & Risk & Pairs & Index $i$ & Index $j$ & Probability \\
\hline $\mathrm{HR}_{1}$ & High & Different & $\begin{array}{l}1 \\
2 \\
3 \\
7 \\
8 \\
9\end{array}$ & $\begin{array}{l}j \in\{2,3,7,8,9\} \\
j \in\{1,3,7,8,9\} \\
j \in\{1,2,7,8,9\} \\
j \in\{1,2,3,8,9\} \\
j \in\{1,2,3,7,9\} \\
j \in\{1,2,3,7,8\}\end{array}$ & $\begin{array}{l}P\left(H_{1}\right)\left(P\left(H_{2}\right)+P\left(H_{3}\right)+\ldots+P\left(H_{9}\right)\right) \\
P\left(H_{2}\right)\left(P\left(H_{1}\right)+P\left(H_{3}\right)+\ldots+P\left(H_{9}\right)\right) \\
P\left(H_{3}\right)\left(P\left(H_{1}\right)+P\left(H_{2}\right)+\ldots+P\left(H_{9}\right)\right) \\
P\left(H_{7}\right)\left(P\left(H_{1}\right)+P\left(H_{2}\right)+\ldots+P\left(H_{9}\right)\right) \\
P\left(H_{8}\right)\left(P\left(H_{1}\right)+P\left(H_{2}\right)+\ldots+P\left(H_{9}\right)\right) \\
P\left(H_{9}\right)\left(P\left(H_{1}\right)+P\left(H_{2}\right)+\ldots+P\left(H_{8}\right)\right)\end{array}$ \\
\hline $\mathrm{HR}_{2}$ & High & Same & $\begin{array}{r}1 \\
2 \\
3 \\
7 \\
8 \\
9 \\
10\end{array}$ & $\begin{array}{r}1 \\
2 \\
3 \\
7 \\
8 \\
9 \\
10\end{array}$ & $\begin{array}{l}P\left(H_{1}\right)^{2} \\
P\left(H_{2}\right)^{2} \\
P\left(H_{3}\right)^{2} \\
P\left(H_{7}\right)^{2} \\
P\left(H_{8}\right)^{2} \\
P\left(H_{9}\right)^{2} \\
P\left(H_{10}\right)^{2}\end{array}$ \\
\hline $\mathrm{LR}_{1}$ & Low & Same & $\begin{array}{l}4 \\
\ldots \\
12\end{array}$ & $\begin{array}{l}4 \\
\ldots \\
12\end{array}$ & $\begin{array}{l}P\left(H_{4}\right)^{2} \\
\ldots \\
P\left(H_{12}\right)^{2}\end{array}$ \\
\hline $\mathrm{LR}_{2}$ & Low & Different & $\begin{array}{l}1 \\
2 \\
\cdots \\
11 \\
12\end{array}$ & $\begin{array}{l}j \notin\{1,2,3,7,8,9\} \\
j \notin\{1,2,3,7,8,9\} \\
\ldots \\
\neq 11 \\
\neq 12\end{array}$ & $\begin{array}{l}P\left(H_{1}\right)\left(1-P\left(H_{1}\right)-P\left(H_{2}\right)-\ldots-P\left(H_{9}\right)\right) \\
P\left(H_{2}\right)\left(1-P\left(H_{1}\right)-P\left(H_{2}\right)-\ldots-P\left(H_{9}\right)\right) \\
\ldots \\
P\left(H_{11}\right)\left(1-P\left(H_{11}\right)\right) \\
P\left(H_{12}\right)\left(1-P\left(H_{12}\right)\right)\end{array}$ \\
\hline
\end{tabular}

For computational efficiency and convenience, we also differentiate between identical and non-identical haplotypes in a diplotype, denoted as strata $\mathrm{HR}_{1}, \mathrm{HR}_{2}, \mathrm{LR}_{1}$, and $\mathrm{LR}_{2}$, and further stratify by the possible indices of the first haplotype we will sample in a pair (index $i$ ). The strata probabilities are derived from the frequencies in table 2. Note that this somewhat time-consuming tabulation step is carried out when a simulation is initialized, and thus has to be invoked only once.

where $C$ is the diplotype of the child at the locus of interest, as before. The genotype(s) of $X$ and effect sizes are unknown, and thus the penetrance $P(D \mid X)$ is simply assumed to have a given value $\pi$. Thus, we can simply write:

$$
\begin{aligned}
p_{C}^{*} & =P(D \mid C)=1-\left[1-\frac{\exp (\alpha+\beta C)}{1+\exp (\alpha+\beta C)}\right] \times(1-\pi) \\
& =1-\frac{1-\pi}{1+\exp (\alpha+\beta C)} .
\end{aligned}
$$

The only change required in comparison to the previous two risk-group model is the alteration of the sampling probabilities to pick a stratum in the first step (the probability $P(S \mid D)$ in equation $4)$. That is, one needs to find the set of parameters $\alpha^{*}, \beta^{*}$ that satisfy the conditions of equation 6 , namely $\operatorname{logit}\left(p_{C}^{*}\right)=\alpha^{*}+\beta^{*} C$, for $C=0$ and $C=1$. Thus, $\alpha^{*}=\operatorname{logit}\left(p_{C=0}^{*}\right)$, and $\beta^{*}=\operatorname{logit}\left(p_{C=1}^{*}\right)-$ $\operatorname{logit}\left(p_{C=0}^{*}\right)$, which yields (see online suppl. materials) the closedform solution:

$$
\alpha^{*}=\log \left[\frac{\exp (\alpha)+\pi}{1-\pi}\right] \text { and } \beta^{*}=\log \left[\frac{\exp (\alpha+\beta)+\pi}{\exp (\alpha)+\pi}\right] .
$$

\section{Results}

We validated our case-parent simulation approach using interaction models of order 1-6 (table 7), for 15 haplotype blocks containing 45 SNPs. In each setting, the loci contributing to the genetic risk were in separate haplotype blocks. We chose the haplotype frequencies such that about $5 \%$ of the population were carriers of the risk genotype combination. For each setting, we varied the risk among the non-carriers using $\alpha=-5$ (corresponding to a risk of $0.7 \%$ ), $\alpha=-3$ (risk: $4.7 \%$ ), $\alpha=-1$ (risk: $27 \%$ ) in the disease-risk model (equation 1). We also altered the odds ratios $(\mathrm{OR})$ in the risk model using $\beta=0(\mathrm{OR}=1)$, $\beta=1(\mathrm{OR}=2.7), \beta=2(\mathrm{OR}=7.4), \beta=3(\mathrm{OR}=20)$. These extreme values were chosen deliberately, as the objective was to validate the trio simulations. We simulated 100 data sets with 1,000 trios for each $\alpha / \beta$ combination. It is noteworthy that it is possible to enumerate the complete 
Table 5. The distributions for the parental haplotype pairs assuming the child has non-identical $(i, j)$ or identical $(i, i)$ haplotypes

\begin{tabular}{cccl}
\hline Stratum & Index 1 & Index 2 & Probability \\
\hline $\begin{array}{l}\text { Child with different haplotypes }(i, j) \\
\text { Parent } 1\end{array}$ & \\
$A_{1}$ & $i$ & $\neq i, \neq j$ & $2 P\left(H_{j}\right)\left(1-P\left(H_{i}\right)-P\left(H_{j}\right)\right)$ \\
$A_{2}$ & $i$ & $j$ & $2 P\left(H_{i}\right) P\left(H_{j}\right)$ \\
$A_{3}$ & $i$ & $i$ & $P\left(H_{i}\right)^{2}$ \\
& & Total $\rightarrow$ & $2 P\left(H_{i}\right)-P\left(H_{i}\right)^{2}$ \\
Parent 2 & & & \\
$B_{1}$ & $j$ & $\neq i, \neq j$ & $2 P\left(H_{j}\right)\left(1-P\left(H_{i}\right)-P\left(H_{j}\right)\right)$ \\
$B_{2}$ & $j$ & $i$ & $2 P\left(H_{j}\right) P\left(H_{j}\right)$ \\
$B_{3}$ & $j$ & $j$ & $P\left(H_{j}\right)^{2}$ \\
& & Total $\rightarrow$ & $2 P\left(H_{j}\right)-P\left(H_{j}\right)^{2}$ \\
\hline
\end{tabular}

Child with identical haplotypes $(i, i)$

Any Parent

$\begin{array}{cccl}C_{1} & i & \neq i & 2 P\left(H_{i}\right)\left(1-P\left(H_{i}\right)\right) \\ C_{2} & i & i & P\left(H_{i}\right)^{2} \\ & & \text { Total } \rightarrow & 2 P\left(H_{i}\right)-P\left(H_{i}\right)^{2}\end{array}$

The strata's probabilities are derived from the frequencies in table 2 .

mating tables, i.e. the trio haplotype pairs and the respective sampling probabilities, only for very limited interaction terms. With this approach, trios under only the first 3 risk-group definitions (table 7) could be simulated. For the other settings, this approached was aborted because of excessive memory requirements ( $>32 \mathrm{~GB}$ ), and the previously described efficient simulation approach was employed.

The validation of the trio simulation method was primarily based on the expected values of the parameter estimates derived via genotypic TDTs of the simulated data sets. For each of the simulated data sets, we derived the pseudo-controls (the possible but unobserved Mendelian realizations given the parental haplotypes) at each of the loci that affected the risk (between 1 and 6 loci, see table 7). Since these loci were chosen in separate blocks, we combined the 3 pseudo-genotypes in random order at each locus into 3 pseudo-controls. For all cases and controls, we then calculated the Boolean genotype combination that defined risk for each of the cases and pseudocontrols (thus, defining carriers and non-carriers), and we used conditional logistic regression with the carrier status as the predictor of interest. However, when using conditional logistic regression to compare cases and pseudo-controls, the expected value of the parameter es-
Table 6. The parental diplotype-pair distribution assuming the child has non-identical $(i, j)$ or identical $(i, i)$ haplotypes

\begin{tabular}{llllll}
\hline Index & Parent 1 & Parent 2 & Factor P Factor C & $\begin{array}{l}\text { Sampling } \\
\text { probability }\end{array}$ \\
\hline \multicolumn{5}{c}{ Child with different haplotypes $(i, j)$} \\
1 & $A_{1}:(i, k)$ & $B_{1}:(j, k)$ & 2 & $1 / 4$ & $1 / 2 P\left(A_{1}\right) P\left(B_{1}\right)$ \\
2 & $A_{1}:(i, k)$ & $B_{2}:(i, j)$ & 2 & $1 / 4$ & $1 / 2 P\left(A_{1}\right) P\left(B_{2}\right)$ \\
3 & $A_{1}:(i, k)$ & $B_{3}:(j, j)$ & 2 & $1 / 2$ & $P\left(A_{1}\right) P\left(B_{3}\right)$ \\
4 & $A_{2}:(i, j)$ & $B_{1}:(j, k)$ & 2 & $1 / 4$ & $1 / 2 P\left(A_{2}\right) P\left(B_{1}\right)$ \\
5 & $A_{2}:(i, j)$ & $B_{2}:(i, j)$ & 1 & $1 / 2$ & $1 / 2 P\left(A_{2}\right) P\left(B_{2}\right)$ \\
6 & $A_{2}:(i, j)$ & $B_{3}:(j, j)$ & 2 & $1 / 2$ & $P\left(A_{2}\right) P\left(B_{3}\right)$ \\
7 & $A_{3}:(i, i)$ & $B_{1}:(j, k)$ & 2 & $1 / 2$ & $P\left(A_{3}\right) P\left(B_{1}\right)$ \\
8 & $A_{3}:(i, i)$ & $B_{2}:(i, j)$ & 2 & $1 / 2$ & $P\left(A_{3}\right) P\left(B_{2}\right)$ \\
9 & $A_{3}:(i, i)$ & $B_{3}:(j, j)$ & 2 & 1 & $2 P\left(A_{3}\right) P\left(B_{3}\right)$ \\
\multicolumn{5}{c}{} \\
\hline \multicolumn{5}{c}{ Child with $i d e n t i c a l$ haplotypes $(i, i)$} \\
1 & $C_{1}:(i, j)$ & $C_{1}:(i, k)$ & 1 & $1 / 4$ & $1 / 4 P\left(C_{1}\right)^{2}$ \\
2 & $C_{2}:(i, i)$ & $C_{1}:(i, k)$ & 2 & $1 / 2$ & $P\left(C_{1}\right) P\left(C_{2}\right)$ \\
3 & $C_{2}:(i, i)$ & $C_{2}:(i, i)$ & 1 & 1 & $P\left(C_{2}\right)^{2}$ \\
& & & Total $\rightarrow$ & $P\left(H_{i}\right)^{2}$ \\
\hline
\end{tabular}

The strata's probabilities are based on the haplotype-pair frequencies in table 5. Two further considerations are important in the derivation of the sampling probabilities. The joint probability for a non-identical pair of diplotypes in the parents has to be multiplied by the factor 2 , since there is no ordering in the parental diplotype pairs (denoted above as Factor P). Further, the number of distinct diplotypes in the 4 'Mendelian' children can be 1,2 , or 4 , depending on the parents' haplotype combinations. This is taken into consideration by multiplying the parents' diplotype probabilities with the respective factor (denoted above as Factor C).

timates is not the $\log \mathrm{OR} \beta$, but the $\log$ relative risk [41]. In our case, the relative risk is given as

$$
\begin{aligned}
R R & =\frac{P\left(D \mid I_{G}=1\right)}{P\left(D \mid I_{G}=0\right)}=\frac{\exp (\alpha+\beta) /[1+\exp (\alpha+\beta)]}{\exp (\alpha) /[1+\exp (\alpha)]} \\
& =\exp (\beta) \times\left[\frac{1+\exp (\alpha+\beta)}{1+\exp (\alpha)}\right]^{-1}
\end{aligned}
$$

and therefore the log relative risk is

$$
\log (R R)=\beta-\log \left[\frac{1+\exp (\alpha+\beta)}{1+\exp (\alpha)}\right] .
$$

The latter term describes the deviation from the log OR $\beta$, and is 0 only if $\beta$ is 0 (i.e. a risk independent of genotypes), and diminishes as $\alpha$ gets small for $\beta \neq 0$. Notice though that in particular for $\alpha=-1$ in our simulation, the 
Table 7. The interactions in the genetic models used to validate the method and algorithm for the case-parent trio simulation

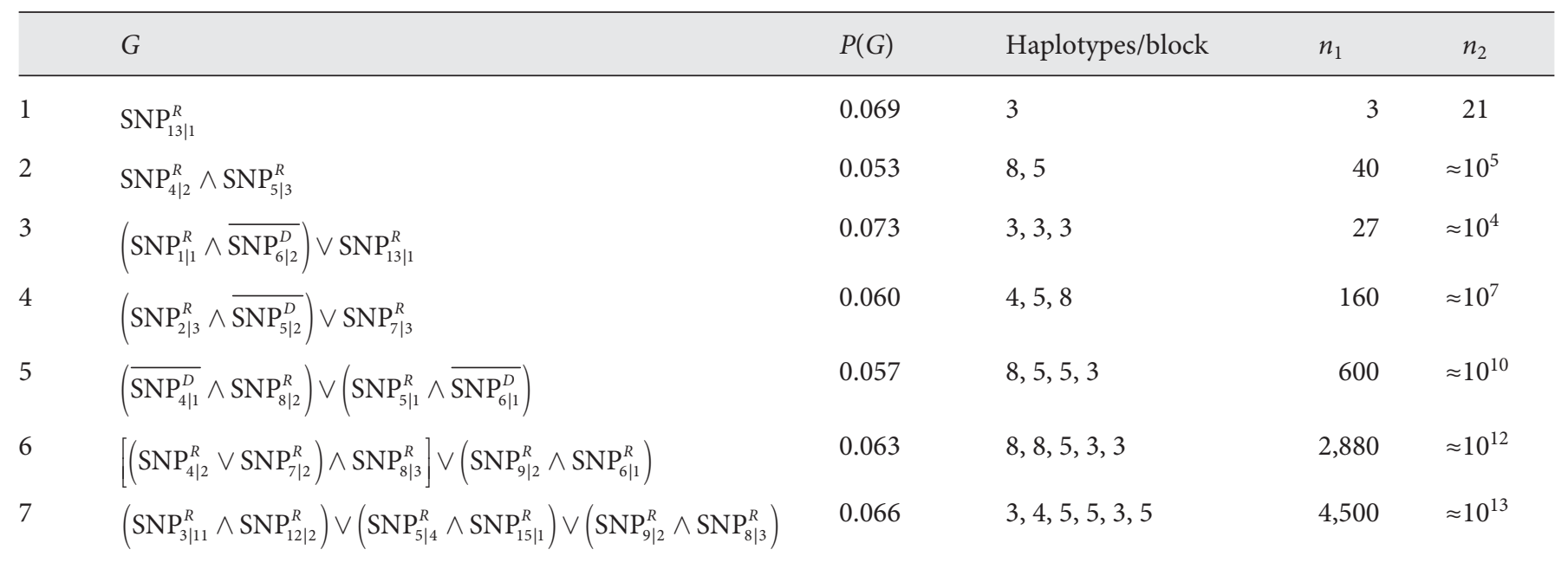

We simulated 15 haplotype blocks containing 45 SNPs based on the above interactions, with various parameters for the diseaserisk model (see text for details). In the above notation, $\mathrm{SNP}_{13 \mid 1}^{R}$ is equal to 1 if the first locus in block 13 has 2 variant alleles. The superscript $D$ denotes an assumed dominant effect (1 or 2 variant alleles), and the horizontal bar denotes the Boolean complement. The symbols $\vee$ and $\wedge$ stand for the Boolean operators 'or' and 'and', respectively. Further, $P(G)$ denotes the proportion of risk carriers in the population. The number of haplotypes in the blocks that contain a locus with disease-risk information was chosen between 3 and 8 . The total number of possible haplotypes is recorded $\left(n_{1}\right)$, as is the number of rows in the respective mating tables $\left(n_{2}\right)$. The efficient simulation method introduced was crucial for interactions $4-8$, as the memory requirements were prohibitive when attempting to calculate the mating table.
Fig. 2. 100 replicates for 1,000 trios were simulated assuming a risk genotype given by the 6-way interaction in table 7 , using various combinations for the parameters $\alpha$ $(-5,-3,-1)$ and $\beta(0,1,2,3,4)$. The exact procedure is described in more detail in the text. The boxplots summarize the $100 \mathrm{pa}$ rameter estimates obtained by using the true risk model as binary predictor in a conditional logistic regression model. The arrows indicate the expected value for the parameters (the log relative risk) as defined in equation 9. The median of each of the parameter estimate sets (shown as a horizontal bar in the center of each box) coincides well with the expected value, thus validating the trio simulation approach. Only the outcome for the 6-way interaction is shown. Results and figures for the other 6 scenarios as indicated in table 7 were identical. Note that the generation of the mating tables is prohibitive for all but the most simple set-ups (examples 1-3 in table 7), and a more efficient approach such as the one in the Methods section has to be employed.

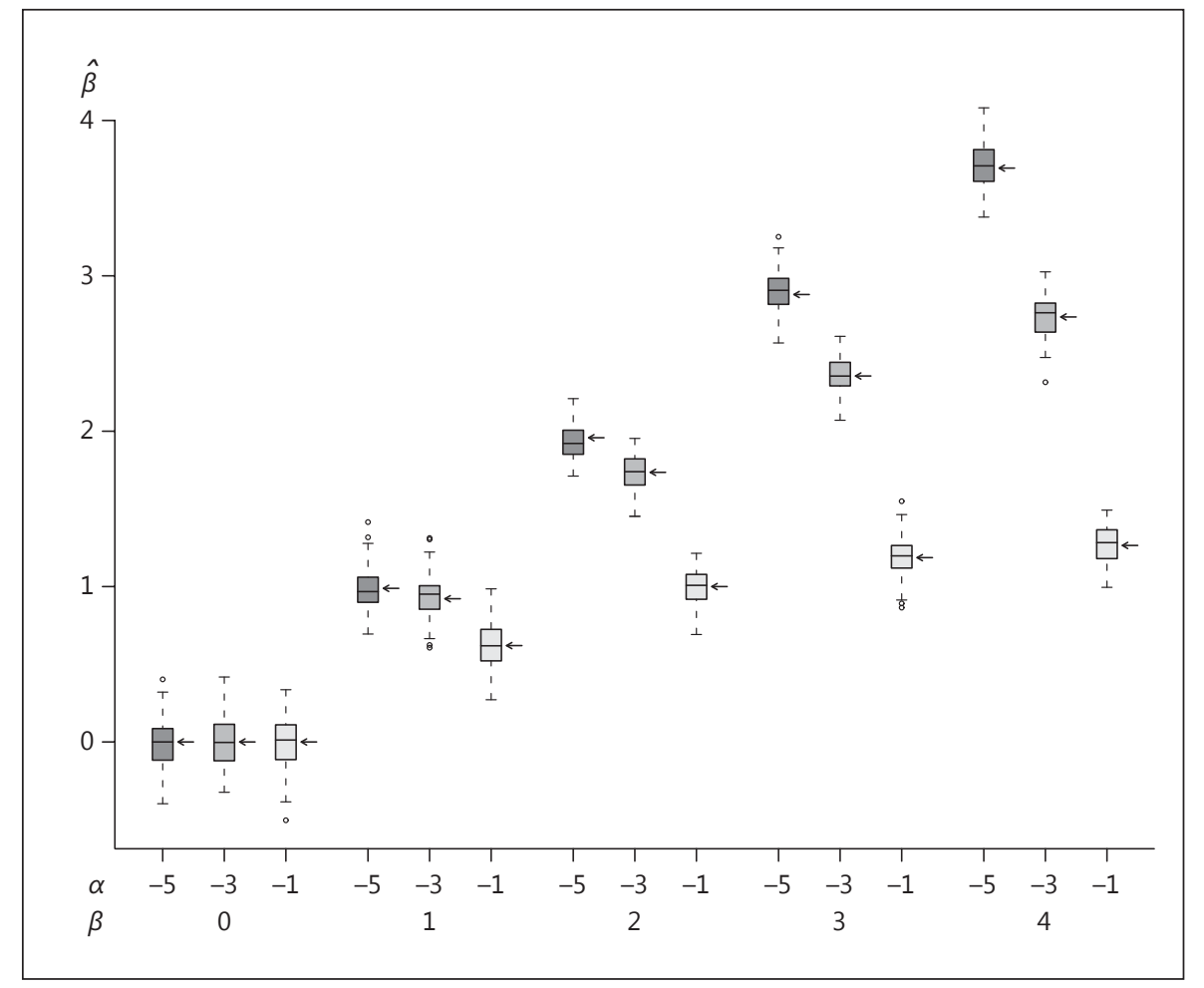




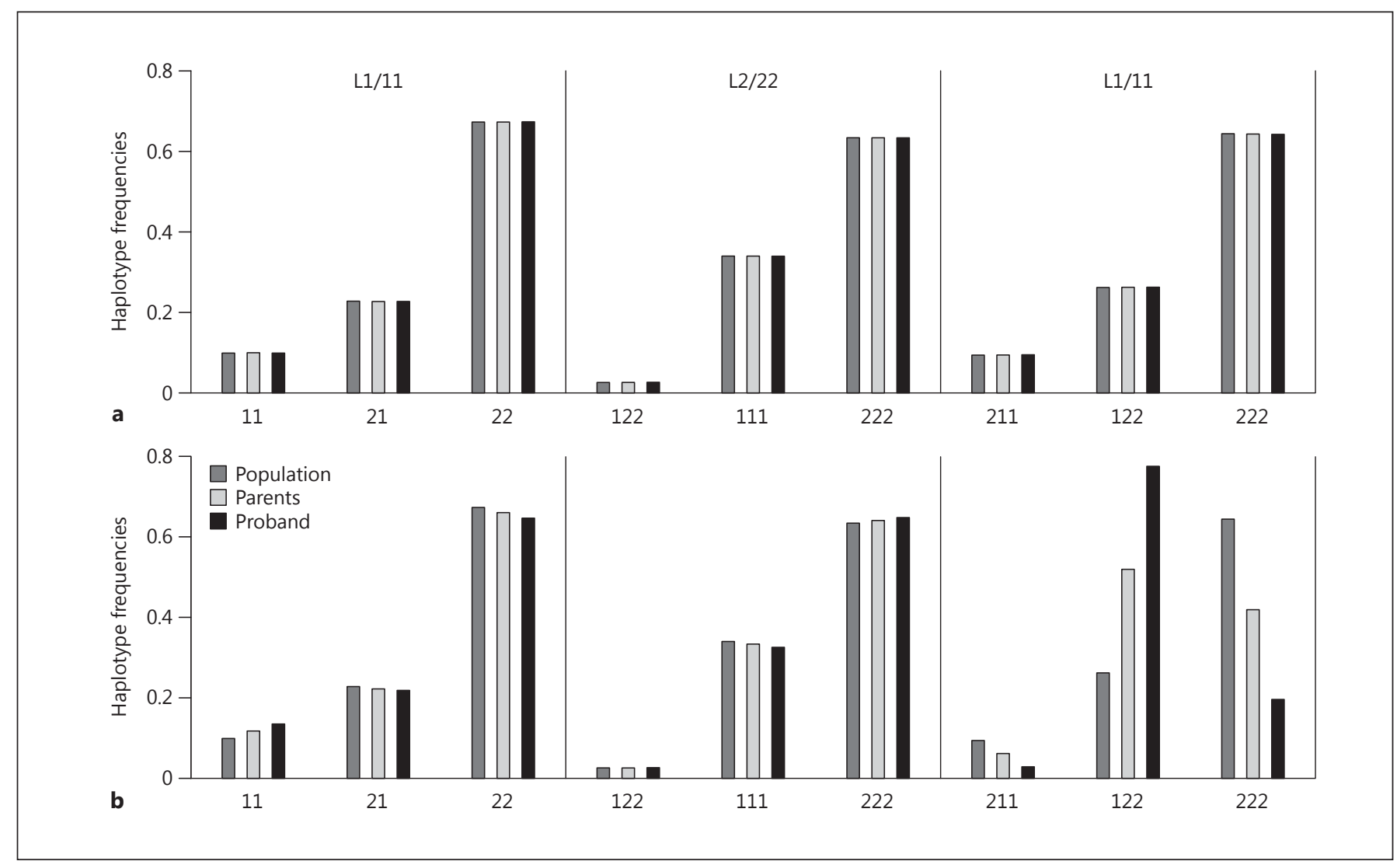

Fig. 3. 100 replicates for 1,000 trios were simulated assuming a risk genotype given by the 3-way interaction

$$
\left(\mathrm{SNP}_{1 \mid 1}^{R} \wedge \overline{\mathrm{SNP}_{6 \mid 2}^{D}}\right) \vee \mathrm{SNP}_{13 \mid 1}^{R}
$$

(table 7, scenario 3), using $\alpha=-5$ and $\beta=0$ (a) and $\beta=4$ (b). The bars show the haplotype frequencies in the 3 blocks that carry disease-risk information (block 1: left, block 6: middle, block 13: right). In each block, we assumed that 3 different haplotypes exist in the population, as indicated on the horizontal axis, where 1 refers to the minor (variant) allele, and 2 refers to the major allele. Thus, individuals with 2 variant alleles at locus 1 in block 13 (L1/11, right) are at higher risk for disease if $\beta>0$, as are subjects that have 2 variant alleles at locus 1 in block 1 (L1/11, left) and at

difference between the log relative risk and the log OR can be substantial (fig. 2). We also validated our procedure for the two-locus genetic heterogeneity model, where additional risk loci are assumed (see online suppl. materials).

We also noticed that, as expected, the haplotype frequencies in the blocks that carry risk information deviate from the population frequencies (fig. 3) when generating case-parent trios. We observed that the haplotypes that contribute to disease risk were sampled more frequently

Efficient Simulation of Epistatic

Interactions in Case-Parent Trios the same time no variant alleles at locus 2 in block 6 (L2/22, middle). For each haplotype in each block, we show the population frequency, the frequency among the parents, and the frequency among the probands. As expected, if there is no association between genotypes and outcome $(\beta=0, \mathbf{a})$, the haplotype frequencies do not differ among parents, offspring, and the population at large. However, we observe vast differences when such an association exists $(\beta=4, \mathbf{b})$. As expected, the only haplotype that can give rise to genotype 11 at locus 1 in block 13 is 122 , and it is greatly enriched in parents and particularly in probands (b, right). The same effect, albeit much less pronounced, is still visible for haplotype 11 in block 1 (b, left), and haplotypes 122 and 222 in block 6 (b, middle).

in our simulations compared to the population at large, at the expense of the other haplotypes in the respective blocks.

\section{Discussion}

Population-based designs such as case-control studies have arguably been the more popular approach for genetic association studies, but nonetheless, family-based 
studies including case-parent designs have been or are currently carried out (for example, the International Consortium to Identify Genes and Interactions Controlling Oral Clefts, http://www.genevastudy.org/). The fact that common variants identified through genome-wide association studies (population- and family-based) typically only account for a limited fraction of reported trait heritability, coupled with recent advances in sequencing technologies, has shifted the paradigm for how to conduct genetic association studies. A large number of sequencing studies are currently carried out, particularly to assess the presence of rare variants with larger effect sizes. For those rare variants, family-based approaches including case-parent trios can be particularly attractive, as disease-causing variants should cluster within families, and departures from independent segregation can be observed.

We devised an efficient algorithm to rapidly simulate such case-parent trios, where the genetic risk is determined via higher-order epistatic interactions. Power to detect epistatic interactions using one or several competing statistical approaches can then be assessed. We validated the algorithm using interactions involving up to 6 SNPs, which, due to computational constraints (i.e. in particular due to memory constraints), cannot be achieved by standard mating table computations. The actual algorithm is implemented as a two-step procedure. First the haplotype tables and the respective sampling probabilities necessary to simulate case-parent trios are generated. This step requires specification of the haplotypes and their respective frequencies, or alternatively to delineate and phase LD blocks from genotype data observed in the population at large, if some population-specific and thus possibly more realistic genotype distributions and LD structures are desired. The second step is then to take random draws based on these tables and probabilities. If multiple simulations under the same parameter settings (e.g. for power calculations) are required, the tables and sampling probabilities only need to be generated once, and an arbitrary number of draws can be taken based on these. This procedure ensures that even if a large number of trios needs to be simulated, results are available almost instantaneously (see online suppl. materials).

The efficiency of our simulation approach is in part owed to the fact that only two risk groups are assumed to exist in the population (carriers and non-carriers), which is used for the calculation of the haplotype frequencies in the blocks that carry information about the disease risk. This seems like a limitation at first, since, in particular for complex diseases, more than a single interaction between some SNPs could be expected to affect the outcome. However, we were able to extend our methods to the 'two-locus' genetic heterogeneity framework [65], allowing for a much more general setting. Under the assumption that the two 'loci' - the specified interaction taken as the first 'locus', the second 'locus' representing all other disease loci that define disease risk - are independent causes of disease, we derived closed-form solutions for the parameters needed in the simulation, and implemented this approach in the open source software R package trio. Other possible models for the interaction term, such as models involving additive genetic effects or continuous environmental covariates, cannot be accommodated in a straightforward manner unless they are dichotomized a priori, since they typically generate more than two risk groups as assumed in our proposed simulation framework. However, this type of interaction complexity is not typically sought for simulations. Instead, we often acknowledge that even complicated simulated models are an over-simplification of the unknown 'truth', and thus using a simple epistatic model, among many imperfect models, is the most interpretable. Nonetheless, any additive or quantitative main effects, not involved in the interaction, can be included in the simulating scenario as long as they are independent of the loci involved in the epistatic interaction, simply by using a trio-specific penetrance $P(D \mid X)$ instead of a constant $\pi$ in equation 6 .

The question also arises whether this approach can be extended further, for example to accommodate other phenotypes than disease indicators. Trios with quantitative traits that depend on epistatic interactions or other genetic models can simply be generated prospectively: i.e. randomly sampling parental haplotypes from the population, generating diplotypes for an offspring, and simulating the parents' and child's phenotypes according to the underlying genetic model. No rejection sampling is needed in this case and the simulation is efficient. For genetic models where the quantitative trait depends on epistatic interactions, it is not obvious how our framework could readily be extended, for example to simulate trios with offspring showing extreme phenotypes, to avoid the less efficient option of a prospective simulation followed by subsetting of individuals based on phenotype. However, the need to simulate such data is also not very obvious. While selecting individuals based on extreme phenotypes has become a popular choice for genome-wide association studies and sequencing studies, study designs are usually based on unrelated individuals [66-69]. Linkage 
studies for quantitative traits using extreme phenotypes have also been employed in the past, but designs such as discordant sib pairs are certainly more common in these settings than trios [70-73].

In summary, we have developed an efficient simulation approach to model higher-order interactions in the case-parent trio design setting that can also accommodate genetic heterogeneity. This method, and associated software, is a useful tool for genetic analysis method development in an era of increasing attention to interactions and re-emerging use of family-based designs.

\section{Acknowledgements}

Support was provided by the Deutsche Forschungsgemeinschaft (SCHW 1508/3-1 to H.S.), the National Institutes of Health (R03 DE021437 to I.R.), and a CTSA grant to the Johns Hopkins Medical Institutions.

\section{References}

1 Ritchie MD, White BC, Parker JS, Hahn LW, Moore JH: Optimization of neural network architecture using genetic programming improves detection and modeling of gene-gene interactions in studies of human diseases. BMC Bioinformatics 2003;4:28.

-2 North BV, Curtis D, Cassell PG, Hitman GA, Sham PC: Assessing optimal neural network architecture for identifying disease-associated multi-marker genotypes using a permutation test, and application to calpain 10 polymorphisms associated with diabetes. Ann Hum Genet 2003;67:348-356.

- 3 Tomita Y, Tomida S, Hasegawa Y, Suzuki Y, Shirakawa T, Kobayashi T, Honda H: Artificial neural network approach for selection of susceptible single nucleotide polymorphisms and construction of prediction model on childhood allergic asthma. BMC Bioinformatics 2004;5:120.

4 Breiman L: Statistical modeling: the two cultures. Stat Sci 2001;16:199-215.

$\checkmark 5$ Lunetta KL, Hayward LB, Segal J, Van Eerdewegh P: Screening large-scale association study data: exploiting interactions using random forests. BMC Genet 2004;5:32.

6 Bureau A, Dupuis J, Falls K, Lunetta KL, Hayward B, Keith TP, Van Eerdewegh P: Identifying SNPs predictive of phenotype using random forests. Genet Epidemiol 2005;28:171182.

7 Chen X, Liu CT, Zhang M, Zhang H: A forestbased approach to identifying gene and genegene interactions. Proc Natl Acad Sci USA 2007;104:19199-19203.

8 Chen CH, Chang CJ, Yang WJ, Chen CL, Fann CSJ: A genome-wide scan using treebased association analysis for candidate loci related to fasting plasma glucose levels. BMC Genet 2003;4(suppl 1):S65.

$\checkmark 9$ Cook NR, Zee RYL, Ridker PM: Tree and spline based association analysis of gene-gene interaction models for ischemic stroke. Stat Med 2004;23:1439-1453.
10 Zhang Z, Zhang S, Wong MY, Wareham NJ, Sha Q: An ensemble learning approach jointly modeling main and interaction effects in genetic association studies. Genet Epidemiol 2008;32:285-300.

11 Hahn LW, Ritchie MD, Moore JH: Multifactor dimensionality reduction software for detecting gene-gene and gene-environment interactions. Bioinformatics 2003;19:376-382.

12 Ritchie MD, Hahn LW, Moore JH: Power of multifactor dimensionality reduction for detecting gene-gene interactions in the presence of genotyping error, missing data, phenocopy, and genetic heterogeneity. Genet Epidemiol 2003;24:150-157.

13 Moore JH: Computational analysis of genegene interactions using multifactor dimensionality reduction. Expert Rev Mol Diagn 2004;4:795-803.

14 Ritchie MD, Motsinger AA: Multifactor di mensionality reduction for detecting genegene and gene-environment interactions in pharmacogenomics studies. Pharmacogenomics 2005;6:823-834.

15 Ritchie MD: Bioinformatics approaches for detecting gene-gene and gene-environment interactions in studies of human disease. Neurosurg Focus 2005; 19:E2.

16 Culverhouse R, Klein T, Shannon W: Detecting epistatic interactions contributing to quantitative traits. Genet Epidemiol 2004;27: 141-152.

17 Culverhouse R, Hinrichs AL, Jin CH, Suarez BK: Gene $\times$ gene and gene $\times$ environment interactions for complex disorders. BMC Proc 2007;1(suppl 1):S72.

18 Kooperberg C, Ruczinski I, LeBlanc ML, Hsu $\mathrm{L}$ : Sequence analysis using logic regression. Genet Epidemiol 2001;(suppl 1):S626-S631.

19 Ruczinski I, Kooperberg C, LeBlanc M: Logic regression. J Comput Graph Stat 2003;12: 475-511.

20 Ruczinski I, Kooperberg C, LeBlanc ML: Exploring interactions in high dimensional genomic data: an overview of logic regression, with applications. J Multivar Anal 2004;90: 178-195.
21 Spielman RS, Ewens WJ: The TDT and other family-based tests for linkage disequilibrium and association. Am J Hum Genet 1996;59: 983-989.

22 Gauderman WJ, Witte JS, Thomas DC: Family-based association studies. J Natl Cancer Inst Monogr 1999;26:31-37.

23 Fallin D, Beaty T, Liang KY, Chen W: Power comparisons for genotypic versus allelic TDT methods with $>2$ alleles. Genet Epidemiol 2002;23:458-461.

24 Laird NM, Lange C: Family-based designs in the age of large-scale gene association studies. Nat Rev Genet 2006;7:385-394.

-25 Spielman RS, McGinnis RE, Ewens WJ: Transmission test for linkage disequilibrium: the insulin gene region and insulin-dependent diabetes mellitus (IDDM). Am J Hum Genet 1993;52:506-516.

26 Laird NM, Lange C: Family-based methods for linkage and association analysis. Adv Genet 2008;60:219-252.

27 Schaid DJ: Likelihoods and TDT for the caseparents design. Genet Epidemiol 1999;16: 250-260.

28 Lunetta KL, Faraone SV, Biederman J, Laird NM: Family-based tests of association and linkage that use unaffected sibs, covariates, and interactions. Am J Hum Genet 2000;66: 605-614

29 Culverhouse R, Suarez BK, Lin J, Reich T: A perspective on epistasis: limits of models displaying no main effect. Am J Hum Genet 2002;70:461-471.

- 30 Lanktree MB, Van der Beek L, Macciardi FM Kennedy JL: PedSplit: pedigree management for stratified analysis. Bioinformatics 2004;20: 2315-2316.

31 Baksh MF, Balding DJ, Vyse TJ, Whittaker JC: A likelihood ratio approach to family-based association studies with covariates. Ann Hum Genet 2006;70:131-139.

32 Baksh MF, Balding DJ, Vyse TJ, Whittaker JC: Family-based association analysis with ordered categorical phenotypes, covariates and interactions. Genet Epidemiol 2007;31:1-8. 
- 33 Kotti S, Bickeboller H, Clerget-Darpoux F: Strategy for detecting susceptibility genes with weak or no marginal effect. Hum Hered 2007;63:85-92.

34 Kotti S, Bourgey M, Clerget-Darpoux F: Power of the 2-locus TDT for testing the interaction of two susceptibility genes. BMC Proc 2007;1(suppl 1):S65.

-35 Cordell HJ: Detecting gene-gene interactions that underlie human diseases. Nat Rev Genet 2009; 10:392-404.

36 Cordell HJ: Estimation and testing of geneenvironment interactions in family-based association studies. Genomics 2009;93:5-9.

- 37 Martin ER, Ritchie MD, Hahn L, Kang S, Moore JH: A novel method to identify genegene effects in nuclear families: the MDRPDT. Genet Epidemiol 2006;30:111-123.

- 38 Martin ER, Bass MP, Gilbert JR, PericakVance MA, Hauser ER: Genotype based association test for general pedigrees: the genotypePDT. Genet Epidemiol 2003;25:203-213.

-39 Li Q, Fallin MD, Louis TA, Lasseter VK, McGrath JA, Avramopoulos D, Wolyniec PS, Valle D, Liang KY, Pulver AE, Ruczinski I: Detection of SNP-SNP interactions in trios of parents with schizophrenic children. Genet Epidemiol 2010;34:396-406.

40 Self SG, Longton G, Kopecky KJ, Liang KY: On estimating HLA/disease association with application to a study of aplastic anemia. Biometrics 1991;47:53-61.

-41 Schaid DJ: General Score tests for associations of genetic markers with disease using cases and their parents. Genet Epidemiol 1996;13: 423-449.

-42 Terwilliger JD, Speer M, Ott J: Chromosomebased method for rapid computer simulation in human genetic linkage analysis. Genet Epidemiol 1993; 10:217-224.

-43 Hampe J, Wienker T, Schreiber S, Nuernberg P: POPSIM: a general population simulation program. Bioinformatics 1998;14:458-464.

44 Excoffier L, Novembre J, Schneider S: SIMCOAL: a general coalescent program for the simulation of molecular data in interconnected populations with arbitrary demography. J Hered 2000;91:506-509.

45 Balloux F: EASYPOP (version1.7): a computer program for population genetics simulations. J Hered 2001;92:301-302.

46 Peng B, Kimmel M: simuPOP: a forward-time population genetics simulation environment. Bioinformatics 2005;21:3686-3687.

47 Peng B, Amos CI, Kimmel M: Forward-time simulations of human populations with complex diseases. PLoS Genet 2007;3:e47.
48 Hoggart CJ, Chadeau-Hyam M, Clark TG, Lampariello R, Whittaker JC, DeIorio M, Balding DJ: Sequence-level population simulations over large genomic regions. Genetics 2007;177:1725-1731.

49 Carvajal-Rodriguez A: GENOMEPOP: a program to simulate genomes in populations. BMC Bioinformatics 2008;9:223.

50 Carvajal-Rodriguez A: Simulation of genes and genomes forward in time. Curr Genomics 2010;11:58-61.

51 Rambaut A, Grassly NC: Seq-Gen: an application for the Monte Carlo simulation of DNA sequence evolution along phylogenetic trees. Comput Appl Biosci 1997;13:235-238.

52 Hudson RR: Generating samples under a Wright-Fisher neutral model of genetic variation. Bioinformatics 2002;18:337-338.

53 Moehle M, Sagitov S: Coalescent patterns in diploid exchangeable population models. J Math Biol 2003;47:337-352.

54 Spencer CCA, Coop G: SelSim: a program to simulate population genetic data with natural selection and recombination. Bioinformatics 2004;20:3673-3675.

55 Gasbarra D, Sillanpaa MJ, Arjas E: Backward simulation of ancestors of sampled individuals. Theor Popul Biol 2005;67:75-83.

56 Mailund T, Schierup MH, Pedersen CNS, Mechlenborg PJM, Madsen JN, Schauser L: CoaSim: a flexible environment for simulating genetic data under coalescent models. BMC Bioinformatics 2005;6:252.

57 Marjoram P and Wall JD: Fast 'coalescent' simulation. BMC Genet 2006;7:16.

58 Hellenthal G, Stephens M: msHOT: modifying Hudson's ms simulator to incorporate crossover and gene conversion hotspots. Bioinformatics 2007;23:520-521.

59 Liang L, Zoellner S, Abecasis GR: GENOME: a rapid coalescent based whole genome simulator. Bioinformatics 2007;23:1565-1567.

60 Ramos-Onsins SE, Mitchell-Olds T: Mlcoalsim: multilocus coalescent simulations. Evol Bioinform Online 2007;3:41-44.

61 Ray N, Currat M, Foll M, Excoffier L: SPLATCHE2: a spatially explicit simulation framework for complex demography, genetic admixture and recombination. Bioinformatics 2010;26:2993-2994.

62 Chiu YF, Liang KY, Pan WH: Incorporating covariates into multipoint association mapping in the case-parent design. Hum Hered 2010;69:229-241.
63 Ainsworth HF, Unwin J, Jamison DL, Cordell HJ: Investigation of maternal effects, maternal-fetal interactions and parent-of-origin effects (imprinting), using mothers and their offspring. Genet Epidemiol 2011;35:19-45.

64 Weinberg CR, Wilcox AJ, Lie RT: A log-linear approach to case-parent-triad data: assessing effects of disease genes that act either directly or through maternal effects and that may be subject to parental imprinting. Am J Hum Genet 1998;62:969-978.

65 Risch N: Linkage strategies for genetically complex traits. I. Multilocus models. Am J Hum Genet 1990;46:222-228.

66 Bamshad MJ, Ng SB, Bigham AW, Tabor HK, Emond MJ, Nickerson DA, Shendure J: Exome sequencing as a tool for Mendelian disease gene discovery. Nat Rev Genet 2011; 12:745-755.

67 Guey LT, Kravic J, Melander O, Burtt NP, Laramie JM, Lyssenko V, Jonsson A, Lindholm E, Tuomi T, BoIsomaa, Nilsson P, Almgren P, Kathiresan P, Groop L, Seymour $A B$, Altshuler D, Voight BF: Power in the phenotypic extremes: a simulation study of power in discovery and replication of rare variants. Genet Epidemiol 2011;35:236-246.

68 Wang K, Li WD, Zhang CK, Wang Z, Glessner JT, Grant SFA, Zhao H, Hakonarson H, Price RA: A genome-wide association study on obesity and obesity-related traits. PLoS One 2011;6:e18939.

-69 Emond MJ, Louie T, Emerson J, Zhao W, Mathias RA, Knowles MR, Wright FA, Rieder MJ, Tabor HK, Nickerson DA, Barnes KC, National Heart, Lung, and Blood Institute (NHLBI) GO Exome Sequencing Project, Gibson RL, Bamshad MJ: Exome sequencing of extreme phenotypes identifies DCTN4 as a modifier of chronic Pseudomonas aeruginosa infection in cystic fibrosis. Nat Genet 2012;44: 886-889.

70 Carey G Williamson J: Linkage analysis of quantitative traits: increased power by using selected samples. Am J Hum Genet 1991;49: 786-796.

71 Risch N, Zhang H: Extreme discordant sib pairs for mapping quantitative trait loci in humans. Science 1995;268:1584-1589.

72 Risch NJ, Zhang H: Mapping quantitative trait loci with extreme discordant sib pairs: sampling considerations. Am J Hum Genet 1996;58:836-843.

73 Abecasis GR, Cookson WO, Cardon LR: The power to detect linkage disequilibrium with quantitative traits in selected samples. Am J Hum Genet 2001;68:1463-1474. 